\title{
Modelo de gestión de calidad para empresas agroindustriales. Caso de estudio: florícola
}

\author{
Modelo de gestión de calidad para empresas agroindustriales. Caso de \\ estudio: florícola
}

Jaime Iván Acosta Velarde. ${ }^{1}$, Sayuri Monserrath Bonilla Novillo. ${ }^{2}$, Lenin Santiago Orozco Cantos. ${ }^{3}$, Edwin Ángel Jácome Domínguez.. ${ }^{4} \&$ Gloria Elizabeth Miño Cascante ${ }^{5}$

\begin{abstract}
.
DOI: https://doi.org/10.33262/cienciadigital.v3i2.446

This article presents a methodology for design and partial implementation of a Management model focuses on Quality and productive improvement through methods that allow identifying critical factors which influence all over floriculture industry processes as case of study. Along with other techniques were applied and let to evaluate all factor one by one. Management Performance rates were calculated on sales process, customer satisfaction due to these factors affect the productivity. In addition, evaluation method was used for choosing suppliers. Value added is required to improve the company performance. Statistical control process is necessary to control the quality and customer satisfaction. Therefore, this methodology can be used at all other agroindustry to get more productivity and get efficient management process.
\end{abstract}

Keywords: Productivity. Process. Quality. Indicators.

\footnotetext{
${ }^{1}$ Escuela Superior Politécnica de Chimborazo, Facultad de Mecánica. Riobamba, Ecuador, ji_acosta@espoch.edu.ec

${ }^{2}$ Escuela Superior Politécnica de Chimborazo, Facultad de Administración de Empresas. Riobamba, Ecuador. smbonilla@espoch.edu.ec

${ }^{3}$ Escuela Superior Politécnica de Chimborazo, Facultad de Mecánica. Riobamba, Ecuador. lsorozco@espoch.edu.ec

${ }^{4}$ Escuela Politécnica Nacional, Departamento de Ingeniería Mecánica. Quito, Ecuador. edwin.jacome@epn.edu.ec

${ }^{5}$ Escuela Superior Politécnica de Chimborazo, Facultad de Mecánica. Riobamba, Ecuador. gmino@espoch.edu.ec 


\section{Resumen.}

El presente artículo muestra una metodología para el diseño e implementación parcial de un modelo de gestión orientada a la calidad y la mejora de la productividad a través de la utilización de métodos y técnicas que permiten identificar factores críticos que inciden en los procesos de una empresa florícola, considerada como caso de estudio, posteriormente se aplicaron técnicas que permiten evaluar cada uno de los factores por medio de indicadores de desempeño de gestión, los cuales han sido utilizados para medir el proceso de ventas, satisfacción del cliente y su efecto sobre la productividad. Adicional, se establece un método de selección y evaluación de proveedores como valor agregado al mejoramiento del desempeño de la organización. Se explica brevemente la aplicación de métodos estadísticos para control de calidad para determinar el nivel de satisfacción del cliente externo. Se deduce por tanto, que esta metodología puede ser aplicado en el ámbito agroindustrial lo cual se traduce en la incorporación de valor agregado en los procesos administrativos de las organizaciones.

Palabras claves: Productividad. Procesos. Indicadores. Calidad

\section{Introducción.}

La ciencia de la ingeniería industrial está orientada al desarrollo e implementación de sistemas integrados que permitan el adecuado uso de los recursos que una empresa dispone. Ésta ciencia utiliza métodos y procedimientos de análisis que contribuyen a mejorar continuamente los procesos utilizados en las organizaciones para lograr sus objetivos estratégicos y alcanzar su visión. La aplicación de sus principios, técnicas y procedimientos en diferentes áreas, hace que todo tipo de organización tanto de producción, manufacturera, agrícola y de servicios la consideren como una herramienta estratégica para incrementar el valor añadido en cada uno de los procesos productivos y administrativos de la empresa.

Se considera una empresa florícola como caso de estudio, para la aplicación de técnicas, métodos y/o procedimientos que faciliten las directrices necesarias para determinar y mejorar su desempeño productivo. La empresa se encuentra ubicada en la provincia de Pichincha, cantón Quito, sector Parque Industrial del Sur, se dedica la producción y comercialización de múltiples variedades de rosas para el mercado internacional y nacional desde 1998, tiempo en el cual ha experimentado fases de introducción de productos y posicionamiento en el mercado.

Existen naciones que se han especializado en la producción agrícola y otras en industria; estas últimas producen productos con continuos incrementos de valor agregado articulados con la mejora continua de los procesos utilizados. Los productos industrializados poseen gran valor agregado en comparación con los productos agrícolas cuyo valor agregado no varía 
significativamente con el tiempo. El desarrollo productivo ecuatoriano se ha caracterizado por la producción de mercancías agrícolas o bienes primarios con poco valor agregado como por ejemplo, es el caso de la producción de flores.

El sector florícola se ha convertido en uno de los más representativos contribuyentes de la economía ecuatoriana debido a que desde la década de los ochenta del siglo anterior han experimentado un crecimiento acelerado ya que las exportaciones han aumentado aproximadamente en promedio hasta un 57\% hasta el año 2009, así como también han constituido uno de los rubros de exportación más importantes del país. (FLACSO-MIPRO)

Las condiciones geográficas del Ecuador son propicias para obtener productos con características únicas en cuanto a calidad, por lo que estas flores son altamente codiciadas a nivel internacional, evidenciando ciertas ventajas que poseen las flores producidas en Ecuador en comparación con las producidas a nivel mundial.

Ciertamente, las condiciones ambientales, así como valles andinos que se extienden a lo largo de la cordillera de los Andes con unas temperaturas adecuadas, vertientes y oferta de mano de obra en el Ecuador, hacen que los costos de producir flores sean más baratos que las producidas en otros países, convirtiéndose en ventajas comparativas para el sector.

Las flores así como el banano se han convertido en factores claves de la economía nacional gracias al apoyo que han recibido por parte del estado a través de la Corporación Financiera Nacional para la producción, comercialización y exportación. Sin embargo, es necesario estudios minuciosos en torno a otros sectores productivos agrícolas para determinar ventajas comparativas y establecer líneas y estrategias competitivas con base en el incremento del valor agregado en los productos y servicios a fin de puedan desarrollarse de forma planificada y reciban el apoyo gubernamental apropiado.

Precisamente la productividad se fundamenta en el aprovechamiento de las ventajas comparativas que posee una industria agrícola, esto incluye el manejo eficiente de los recursos utilizados en los distintos procesos que integran la organización así como una gestión eficiente de los procesos que están relacionados con la producción. Sin embargo, uno de los problemas más importantes de éste producto es que no constituye una mercancía elaborada por lo que el valor agregado es bajo y que puede verse afectada por la reducción de la demanda provocado por las crisis económicas de los países compradores.

Se destaca que los procesos de apoyo contribuyen de manera significativa la productividad de estos productos y por lo tanto el enfoque de las continuas mejoras debe estar orientado a generar cambios importantes como el incremento de valor agregado sobre aquellos procesos a fin de incrementar el desempeño de la empresa. Para comprender mejor el desempeño de las empresas florícolas es necesario conocer aspectos importantes que forman parte del proceso de la empresa.

Los aspectos claves del área de Producción tienen una incidencia significativa en el precio final y calidad del producto:

- Sistemas de Producción

- Variedades productivas

- Propagación

- Cultivo en General. 
- Elección del cultivo

- Post-cosecha

- Logística Interna

- Logística Externa

Otro aspecto fundamental es la comercialización y la selección de proveedores debido a que tiene como propósito incrementar el mercado internacional, generando negociaciones confiables entre el comprador, la empresa y proveedor; y si una de las partes falla, la cadena de producción y de valor se verá fuertemente afectada.

Según Carro (2010), la necesidad de mejorar los procesos de producción en industrias que elaboran productos alimenticios exige la búsqueda, el desarrollo y la utilización de nuevas estrategias de gestión, así como herramientas enfocadas a diseñar u optimizar métodos para que sean eficientes y eficaces con el fin de mejorar la productividad y la satisfacción de los clientes.

La identificación de los procesos juega un papel importante para el establecimiento de modelos de gestión enfocada a la calidad. En la empresa florícola existen diferentes tipos de procesos, de acuerdo al rol que desempeñan. A continuación se presenta una breve definición de $\operatorname{los}$

mismos:

Los Procesos Estratégicos: Son los que proporcionan directrices a los demás procesos, para que se pueda lograr la visión de la empresa. Estos procesos comprenden la planificación estratégica y la gestión de la organización. (Runger, 2011)

Gómez, O. (2011) en su publicación explica que se deben establecer estrategias para el control y minimización de costos, orientadas a incrementar el desempeño del recurso humano, mejorar los niveles de producción y actualizar la tecnología aplicada a los procesos.

La norma ISO 9001:2008 promueve la adopción de un enfoque basado en procesos cuando se desarrolla, implementa y mejora la eficacia de un sistema de gestión de la calidad, para aumentar la satisfacción del cliente mediante el cumplimiento de sus requisitos (Verovides $\&$ Michelena, 2013)

El beneficio potencial proviene de la mejora de los procesos en términos de ganancias productivas, reducción de costos y pérdidas, eliminación de problemas en los procedimientos, mejora del control de la gestión, mejora de la calidad y la eficacia (Lafuente, 2009)

Los Procesos Operativos o misionales: Son aquellos que impactan directamente sobre la satisfacción del cliente y cualquier otro aspecto de la misión. Son procesos operativos los de investigación y desarrollo, producción y comercialización. (Runger, 2011)

Procesos de soporte: Son procesos que no están ligados directamente a la misión de la organización, pero resultan necesarios para que los procesos misionales y estratégicos puedan cumplir sus objetivos. Son procesos transversales a toda la organización tales como compras, gestión financiera, gestión de talento humano, gestión de ambiental y evaluación y mejoramiento de la gestión. Figura 1. (Runger, 2011) 


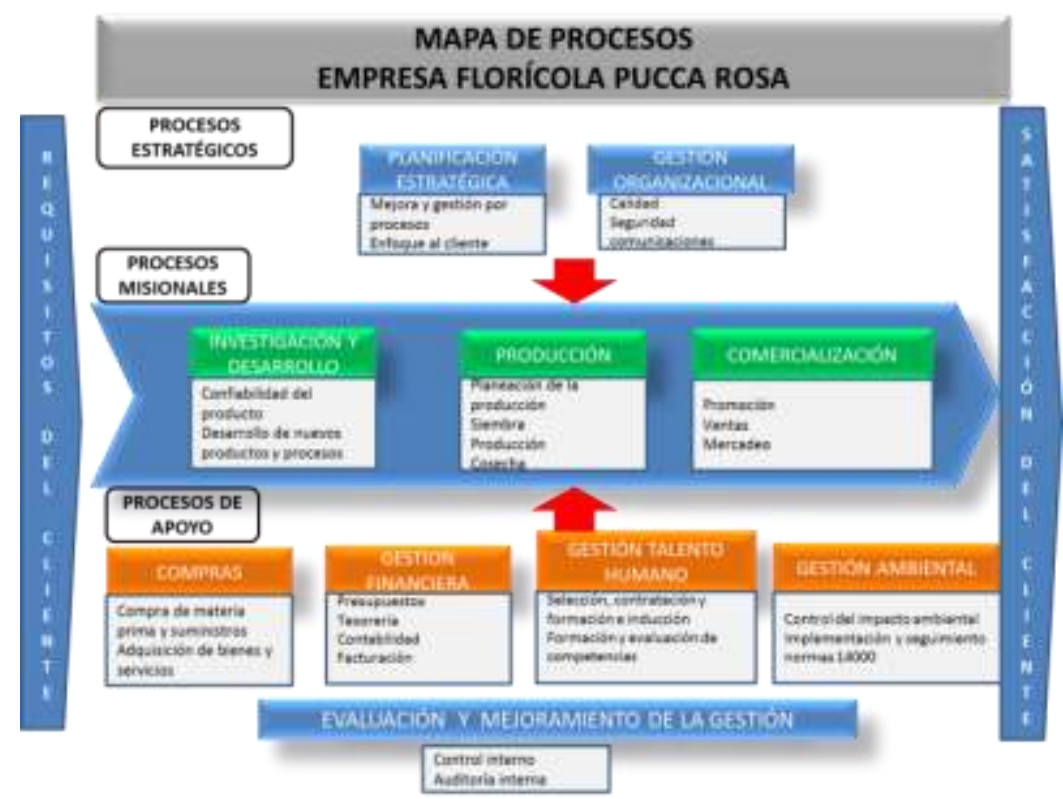

Figura 1. Diagrama de bloques de los procesos de la florícola

Fuente: Runger, 2011.

\section{Metodologi}

\section{Análisis de los procesos claves.}

La investigación parte del análisis de los procesos que constituyen el conjunto de actividades que se interrelacionan para funcionar como un todo encaminadas a lograr un resultado final; sin embargo, se ven afectados por la presencia de inconsistencias o inconformidades que pueden definirse como un incumplimiento de un requisito o como las fallas en la ejecución de los procesos de una organización que busca predecir algo a futuro para generar alternativas de mejora.

Para el estudio se han considerado cinco no conformidades que pueden presentarse en el sistema de gestión de calidad de la empresa florícola.

1. Variabilidad en los procesos

2. Insatisfacción del cliente

3. Perdida de ventas

4. Altos costos de producción

5. Características de calidad de la flor inconsistentes

Se diseña una matriz de clasificación jerárquica aplicada a los problemas con el fin de clasificarlos según su importancia. Esta técnica facilita la identificación de los dos problemas críticos más relevantes en la florícola. Se supone que los datos obtenidos son los resultados 
de decisiones tomadas por un consenso por parte de los directivos y ejecutivos de la empresa a nivel estratégico, con el propósito de determinar aquellos factores que influyen en el desempeño organizacional y ponen en riesgo su permanencia en el mercado. La matriz de priorización o de clasificación jerárquica permite ordenar, de acuerdo a su importancia, los factores identificados como críticos para trazar planes de acción concretos respecto a los problemas enunciados.

Tabla 1. Matriz de clasificación jerárquica.

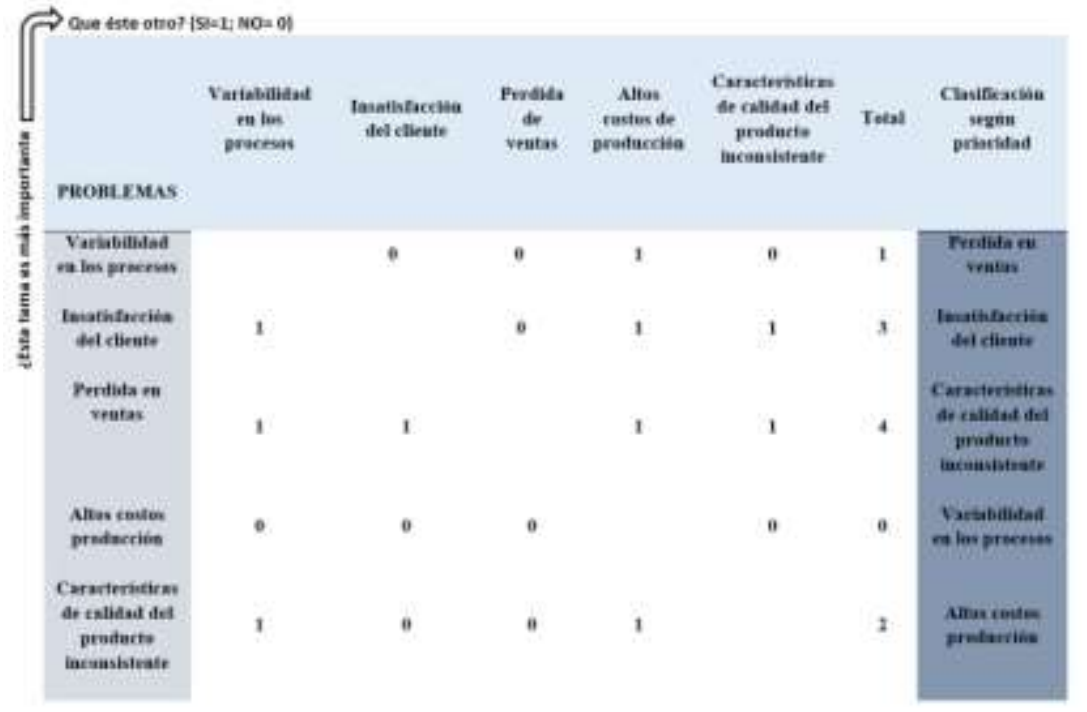

Fuente: Elaboración propia.

De acuerdo a la matriz de clasificación jerárquica, la perdida de ventas afecta directamente la rentabilidad del negocio, a los accionistas y empleados y por ende a toda la organización y actores relacionados. La insatisfacción del cliente es otro factor que incide en el incumplimiento del principio de la calidad enfocado al cliente, esto requiere de todo el esfuerzo posible para satisfacer las necesidades y exceder las expectativas, con el fin de mantener la fidelidad del cliente y que la empresa perdure en el tiempo. (Render, 2009)

\section{Indicadores de desempeño.}

Después de haber identificado aquellos factores críticos que evidentemente influyen en el desempeño de la empresa, precisa establecer indicadores que permitan evaluar el grado de cumplimiento respecto a lo esperado.

A continuación se presentan los indicadores que hacen posible la medición del proceso de ventas así como de la satisfacción del cliente. 
Tabla 3. Indicadores de desempeño: Satisfacción del cliente

\begin{tabular}{|c|c|c|}
\hline $\begin{array}{l}\text { FACTOR } \\
\text { CLAVE }\end{array}$ & INDICADOR & PROCEDIMIENTO DE CÁLCULO \\
\hline \multirow[t]{4}{*}{$\begin{array}{l}\text { Servicio } \\
\text { al cliente }\end{array}$} & $\begin{array}{l}\text { Calidad de } \\
\text { servicio. }\end{array}$ & $\begin{array}{l}\text { Escalas de actitud: Percepción del } \\
\text { cliente sobre un servicio recibido. } \\
\text { Categorias: } \\
\text { Muy satisfactorio (5) } \\
\text { Satisfactorio (4) } \\
\text { Bueno(3) } \\
\text { Regular (2) } \\
\text { Malo(1) }\end{array}$ \\
\hline & $\begin{array}{l}\text { Cumplimiento } \\
\text { entrega }(\%)\end{array}$ & $\frac{\text { tiempo acordado }}{\text { tiempo real de entrega }}+100$ \\
\hline & $\begin{array}{l}\text { Cumplimiento } \\
\text { garantias }(\%)\end{array}$ & $\frac{\text { garantias cumplidas }}{\text { garantias totales }}+100$ \\
\hline & $\begin{array}{l}\text { Atención } \\
\text { reclamos }(\%)\end{array}$ & $\frac{\text { quejas resueltas }}{\text { quejas totales }} * 100$ \\
\hline Producto & $\begin{array}{l}\text { Calidad del } \\
\text { producto }(\%)\end{array}$ & $\begin{array}{c}\text { Producto vendido - producto devuelto } \\
\begin{array}{c}\text { Producto vendido } \\
+100\end{array}\end{array}$ \\
\hline
\end{tabular}

Fuente: Elaboración propia.

Tabla 2. Indicadores de desempeño: Ventas

\begin{tabular}{|c|c|c|}
\hline $\begin{array}{l}\text { FACTOR } \\
\text { CL.AVE }\end{array}$ & INDICADOR & PROCEDIMENTO DE CÁLCULO \\
\hline \multirow{4}{*}{$\begin{array}{l}\text { FUERZA DE } \\
\text { VENTAS } \\
\text { (Vendedores) }\end{array}$} & Ventas por cliente (Sicliente) & $\frac{\text { Ventas realizadas }}{\text { clientes visitados }}$ \\
\hline & Eficacia vendedor ( $\%$ ) & $\frac{\text { Ventas realizadas }(\$)}{\text { Ventas totales }(\$)}+100$ \\
\hline & Eficiencia (\%) & $\frac{\text { Ventas realizadas }(\$)}{\text { Ventas esperadas }(\$)} \cdot 100$ \\
\hline & Productisidad $(\%)$ & $\frac{\text { Incremento Ventas }(\$)}{\text { Costo mano de obra }(\$)} * 100$ \\
\hline \multirow{4}{*}{$\begin{array}{l}\text { PENETRACIÓN } \\
\text { EN } \\
\text { MERCADO }\end{array}$} & Incremento clientes (\%) & $\frac{\text { Clientes nuevos }}{\text { Total clientes }} \cdot 100$ \\
\hline & Impacto Publicidad (\%) & $\frac{\text { Incremento ventas }}{\text { Costo publicidad }} \cdot 100$ \\
\hline & Ventas establecimiento (\%) & $\frac{\text { ventas/establecimiento }}{\text { Ventas totales }}$ \\
\hline & Ventas por producto (7a) & $\frac{\text { Ventas/producto }(\$)}{\text { Ventas totales }(\$)} \cdot 100$ \\
\hline
\end{tabular}

Fuente: Elaboración propia. 


\section{Planeación de los objetivos de calidad orientados a mejorar los factores críticos.}

Con base en la identificación de los factores críticos, la creación de los objetivos debe ser claro, verificable, medible en el tiempo y sobre todo realizable.

Objetivo 1: Lograr un incremento en ventas de rosas en un $20 \%$ al término del primer semestre del año en curso, en relación con el último semestre del año anterior, a nivel internacional.

Objetivo 2: Lograr la satisfacción del cliente en al menos el 95\% con rosas que cumplan parámetros de calidad internacional y con personal altamente calificado durante el primer trimestre del año en curso.

\section{Plan de cumplimiento de los objetivos}

Para el desarrollo del plan de cumplimiento de los objetivos con respecto a los problemas planteados, se analizaron las posibles causas a través de diagramas árbol de causas y se estableció un plan de actividades en la matriz de cumplimiento de objetivos.

\section{Plan de acción basado en la identificación de causas que provocan pérdida de ventas.}

Según la figura $\mathrm{N}^{\circ} 3$, se establecen las causas probables que influyen en las pérdidas en ventas, de las cuales, se consideran dos causas principales: Producto y personal, las mismas que sirven de base para desarrollar actividades que permitan cumplir con el objetivo principal orientado a incrementar las ventas de rosas de exportación en un $20 \%$ en el $1^{\circ}$ semestre del 2015 .

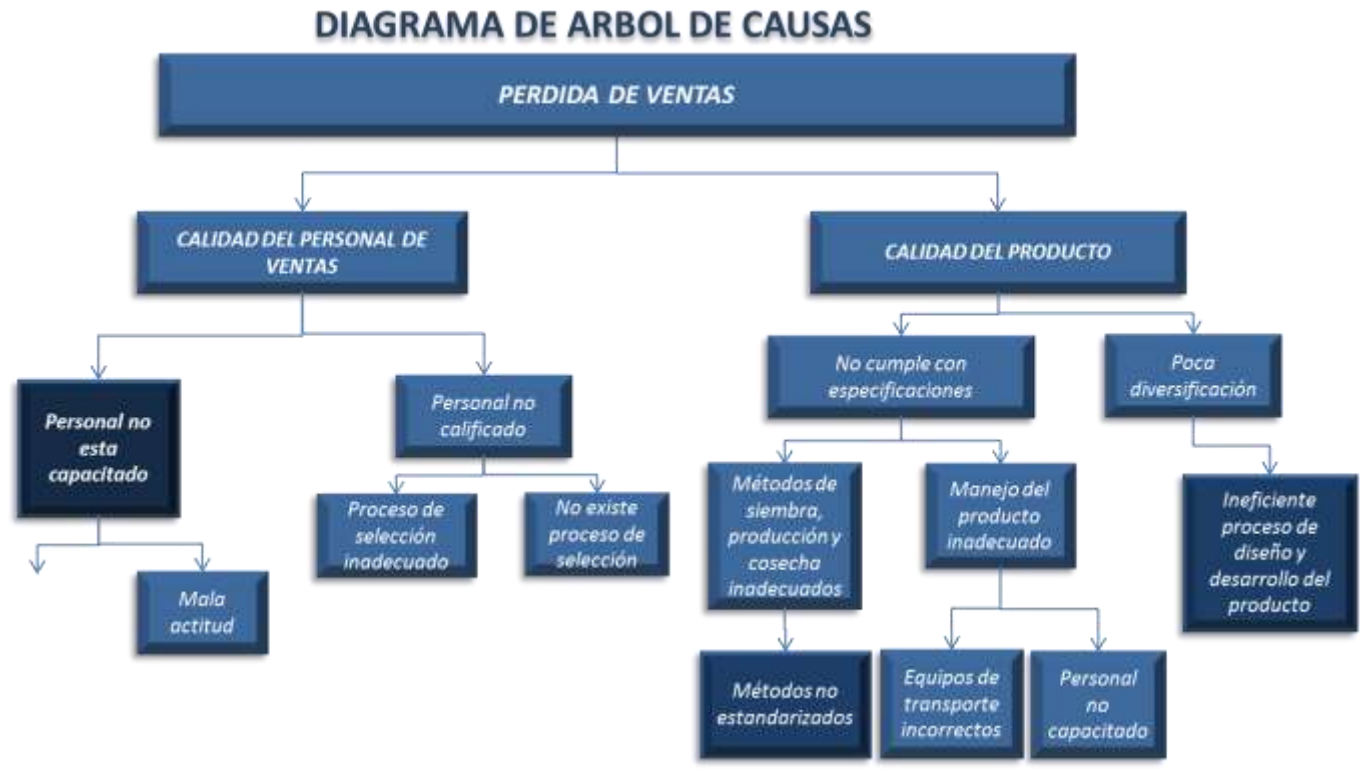

Figura 3. Diagrama árbol de causas: Pérdida en ventas

Fuente: Elaboración propia. 
En la Tabla 4, se han elaborado los planes de mejora a llevarse a cabo para lograr un incremento en ventas con sus respectivas actividades, las mismas que permitirán alcanzar el objetivo planteado en el plazo estipulado. Se orienta a mejorar tres aspectos importantes: publicidad, promoción, personal de ventas calificado y diversificación de rosas de mayor resistencia y calidad.

Tabla 4. Matriz de plan de cumplimiento: Incremento ventas de rosas de exportación

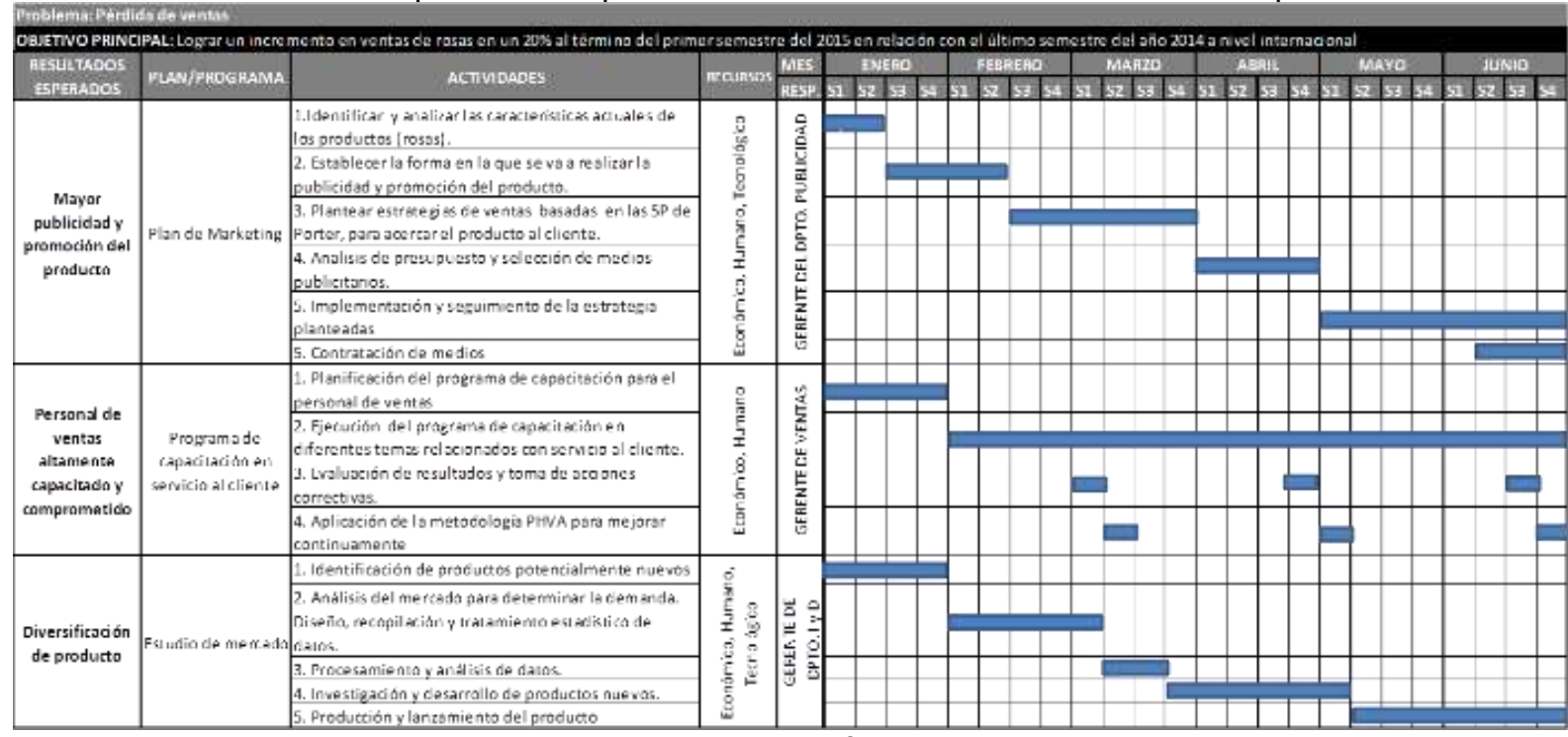

Fuente: Elaboración propia

Plan de acción basado en las causas identificadas que generan el problema insatisfacción del cliente.

De acuerdo con la Figura 4, se identifican causas probables que afectan la satisfacción del cliente. Se asume que las principales causas están relacionadas con la calidad del producto y la calidad de atención al cliente por parte de los empleados, y sobre las cuales habrá que desarrollar planes de acción que minimicen o eliminen su influencia.

DIAGRAMA CAUSA-EFECTO.

PROBLEMA: INSATISFACCIÓN DEL CLIENTE

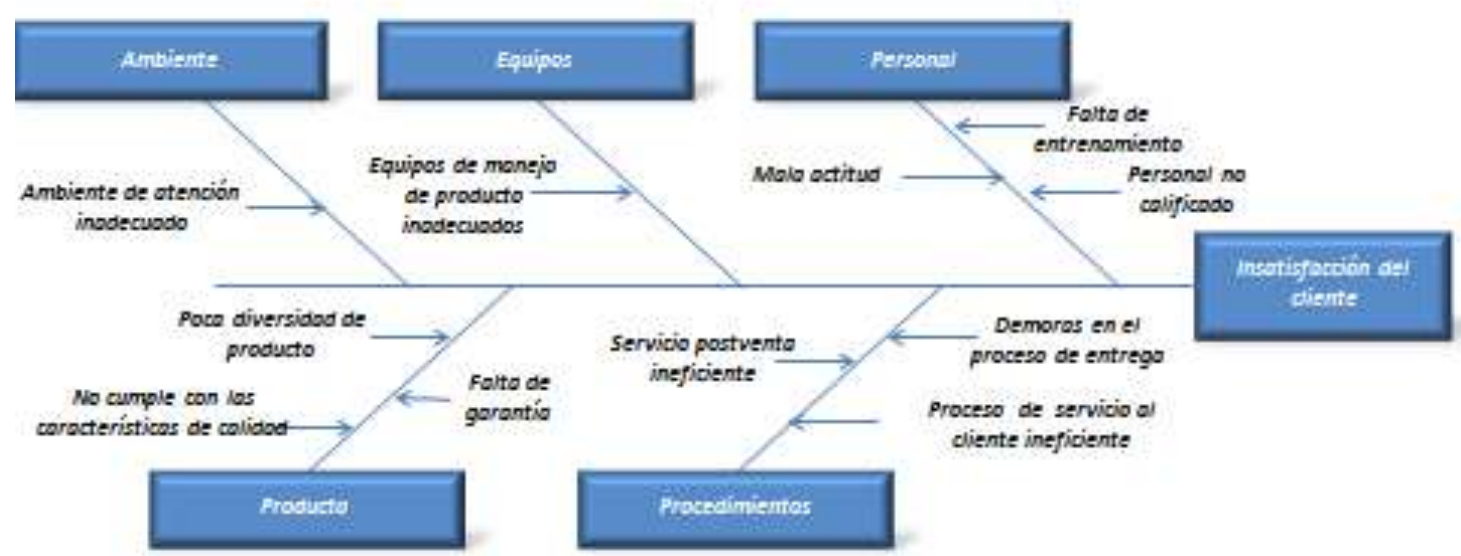

Figura 4. Diagrama causa efecto: Insatisfacción del cliente.

Fuente: Elaboración propia 
Para evaluar el cumplimiento de los planes se requiere la formulación de indicadores de desempeño y el diseño de instrumentos de medición de actitud como las escalas de actitud, encuestas, entrevistas, etc. para la obtención de información del cliente.

Tabla 5. Matriz de cumplimiento: Satisfacción del cliente

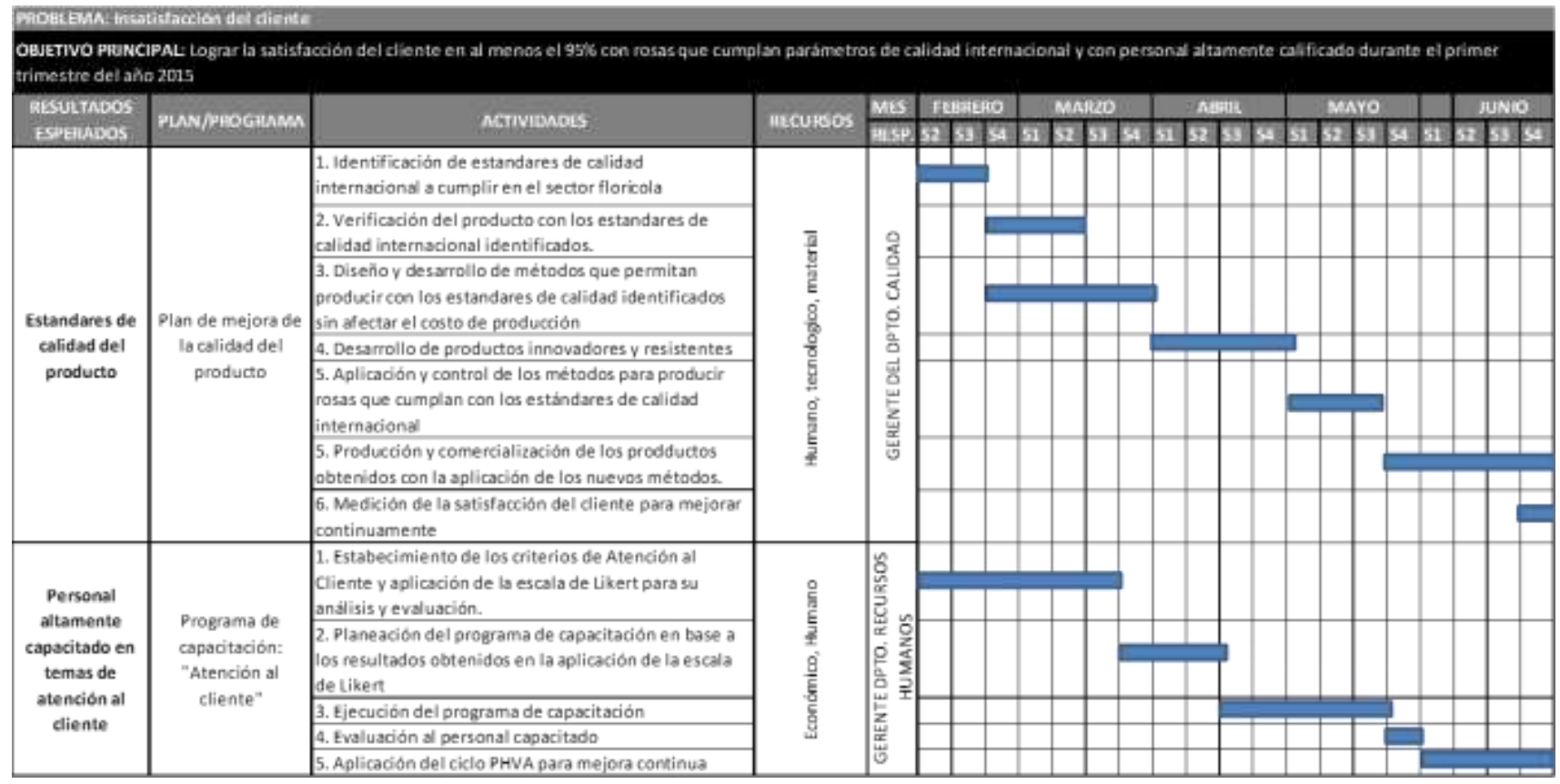

Fuente: Elaboración propia

\section{Elaboración de un instructivo para la evaluación y selección de proveedores}

Tiene como objetivo definir lineamientos que permitan a la empresa florícola seleccionar y evaluar proveedores siguiendo los requisitos contemplados en el Sistema de Gestión de Calidad de los procesos de apoyo de la empresa.

Su Alcance aplica para las etapas de selección y evaluación de proveedores de materia prima, insumos, equipos, maquinaria y/o servicios que contrate la empresa florícola. (Caselles, 2008)

\section{- Selección de proveedores:}

La selección de proveedores para la adquisición bienes y/o servicios es responsabilidad del departamento de adquisiciones el cual deberá tener en cuenta los siguientes criterios de selección para la contratación:

- Costo (Precio más favorable): El proveedor cuenta con la oferta más económica que satisface los requisitos establecidos para la adquisición de bienes y/o servicios. 
- Características técnicas: Proveedor único en el mercado con capacidad de prestar u ofrecer bienes y/o servicios con características específicas requeridas por la empresa.

- Garantía: Se mide el tiempo y modalidad de garantía que el proveedor de los bienes y/o servicios oferte a la empresa.

- Experiencia: El proveedor cuenta con experiencia suficiente en la ejecución de contratos con empresas similares.

- Puntuación de los criterios de selección.

Cada uno de los criterios de selección se evalúa con puntuación dependiendo de la ponderación del criterio evaluado descrito en la tabla $\mathrm{N}^{\circ} 6$.

Tabla 6. Puntuación de los criterios de selección

\begin{tabular}{|c|c|c|c|c|}
\hline CRITERIO & PONDERACION & BAJO & MEDIO & AITO \\
\hline costo & 40 & $\begin{array}{l}\text { Ofereate cos alto costo se } \\
\text { le asigna: } 20 \\
\text { puntes }\end{array}$ & $\begin{array}{l}\text { Ofereate segundo ea } \\
\text { conto recibe: } 30 \text { puntos }\end{array}$ & $\begin{array}{l}\text { Oferente ef costo rids } \\
\text { ecooómico recibe: } \\
40 \text { pantos }\end{array}$ \\
\hline $\begin{array}{c}\text { CARACTERISTICAS } \\
\text { TEECNTCAS }\end{array}$ & 30 & $\begin{array}{l}\text { Si no curiple se le asigna: } \\
\text { e puntos }\end{array}$ & $\begin{array}{l}\text { Si cumple tobalments } \\
\text { recibe: } \\
20 \text { pantes }\end{array}$ & $\begin{array}{l}\text { Si ofinece curacteristicas } \\
\text { técaicas superioces a lo } \\
\text { solicinado recibe } 30 \\
\text { pantos }\end{array}$ \\
\hline GARAVTIA & 20 & $\begin{array}{l}\text { Si no ofrece gurantia se ie } \\
\text { asigna: } \\
\text { opuatos }\end{array}$ & $\begin{array}{l}\text { Si ofrece } 1 \text { abo de } \\
\text { gurautia para equipos y } 3 \\
\text { meses minimo para } \\
\text { insamos recibe: } \\
10 \text { puotos }\end{array}$ & $\begin{array}{l}\text { Si offece } 2 \text { o mits atios } \\
\text { pars equipos y mits de } 3 \\
\text { meses para partes } \\
\text { ingumes, ademis ofiece } \\
\text { otrog valores agregados } \\
\text { recibe: } 20 \text { punates }\end{array}$ \\
\hline EXPERIENCLA & 10 & $\begin{array}{c}\text { Si no fiene } 2 \text { ados minimo } \\
\text { en el mercado se le } \\
\text { asigna: }\end{array}$ & $\begin{array}{l}\text { Si tiene } 2 \text { a } 3 \text { adios en el } \\
\text { mercado recibe: } \\
5 \text { puntos }\end{array}$ & $\begin{array}{l}\text { Si tiene mis de } 3 \text { ados en } \\
\text { el mercado recibe: } 10 \\
\text { pantos }\end{array}$ \\
\hline
\end{tabular}

Fuente: Castillo, M. (2011)

\section{- Valoración de los criterios de selección.}

- Para la selección se requiere que el proveedor cumpla con un mínimo de 60 puntos

- Se escoge al proveedor que tenga el mayor puntaje

- Se inicia la fase contractual

\section{- Evaluación de proveedores.}

Una vez que el proveedor seleccionado cumpla con la entrega de los bienes y/o servicios contratados por parte de la empresa, el departamento de adquisiciones estructura la evaluación del proveedor en dos aspectos: evaluación del plazo de entrega y evaluación de la calidad del bien y/o servicio entregado por el proveedor. 
Los aspectos "plazo de entrega" y "calidad del bien y/o servicio" se los puede evaluar inmediatamente cuando la empresa recibe los bienes y/o servicios contratados y tienen un peso asignado de 0,35 y 0,65 respectivamente de acuerdo a la importancia que tienen para la empresa. (Pereiro, 2005)

\section{- Procedimiento para la evaluación del proveedor.}

El proceso de evaluación inicia con los proveedores seleccionados a los cuales se los califica de acuerdo a su cumplimiento en función de los dos aspectos establecidos por la empresa con los tres criterios que se detallan en la Tabla 7.

Tabla 7. Calificación de los criterios

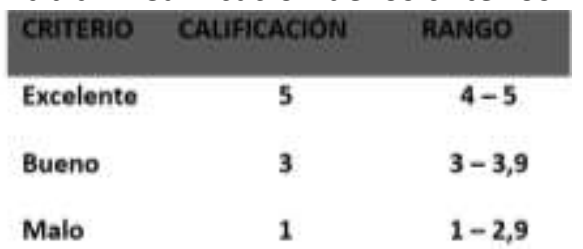

Fuente: Elaboración propia

Posteriormente se multiplica la calificación asignada a cada proveedor con la ponderación correspondiente a cada aspecto (plazo de entrega y calidad del bien y/o servicio) obteniéndose una calificación ponderada. Para cada proveedor se suman las calificaciones ponderadas y dependiendo del rango en el que se encuentre el resultado, se categoriza al proveedor como: excelente, bueno o malo.

\section{Aplicación del instructivo a proveedores críticos de la empresa.}

\section{- Selección de proveedores}

Con el fin de lograr la efectividad en la relación entre la empresa florícola y los proveedores, se establece un método que permitirá identificar a aquellos que cumplan los requerimientos de la empresa en cuanto a parámetros de calidad y eficiencia. Entre los proveedores que requiere la empresa florícola se puede mencionar aquellos que brindan insumos agrícolas, productos químicos, equipos de protección industrial, productos para el empaquetado, entre otros.

Se toma en cuenta cinco proveedores para el proceso de selección y con base a sus ofertas presentadas se los puntúa de acuerdo a los cinco criterios identificados por la empresa para la selección, como lo indica la Tabla 8.

A continuación se consideran los siguientes proveedores 
- Proveedor 1: AMD

Proveedor de insumos para plantaciones y equipos de trabajo. Dedicado a la distribución de

equipos de seguridad así como insumos agrícolas para el sector florícola.

AMD comercializa más de 500 productos en: Ecuador, China, Corea del Sur, Estados Unidos, Canadá, Colombia, Chile, Suecia, Alemania, Rusia e Italia. Los principales productos que comercializa la compañía son: guantes y ligas de caucho con la marca Master; mascarillas y cascos 3M, material de embalaje, láminas de papel, mallas plásticas, grapas industriales, plástico invernadero, cintas adhesiva, tijeras industriales de otras marcas.

\section{- Proveedor 2: DR Global Service}

Proveedores de papel y cartón para el sector florícola. Empresa enfocada al abastecimiento de cajas de cartón corrugado para el sector florícola, con entrega inmediata y a los mejores precios.

\section{- Proveedor 3: Proveedor de papel cartón y cartulina Industrias Alfa}

Se dedica a la transformación del papel, cartón y cartulina en cajas plegadizas, láminas microcorrugadas y una diversidad de productos para la industria farmacéutica, florícola, alimenticia

publicitaria.

- Proveedor 4: Proveedores de suministros SUMIFLOR CIA LTDA

Venta de suministros para agricultura y flores. Está dirigido a las fincas florícolas del país. Cuenta con productos para satisfacer la demanda del mercado agrícola, lechero y florícola, herramientas agrícolas, material de laboratorio, plásticos, tela de sombra, calzado industrial, ropa de trabajo, ropa térmica, trajes de fumigación, protección industrial, material de embalaje, riego, fumigación, equipos de medición, etc.

- Proveedor 5: Proveedores de gavetas cerradas y caladas GAVETA FLOR CIA LTDA.

Proveedor de gavetas cerradas y caladas de varios tamaños para empresas de agroindustria como florícolas, camaroneras, plantaciones en general, así como de huacales para avícolas, pallets, botas de caucho con protección industrial, baldes, tachos de reciclaje, geo membranas, cajoneras, sillas, mesas y todo producto de plástico industriales.

\section{Resultados.}

Los resultados demuestran que los proveedores que cumplen con la condición son: el proveedor AMD, el proveedor DGS y el proveedor INDUSTRIAS ALFA con los cuales se negociarán las compras. 
Tabla 8. Matriz de selección de proveedores

\begin{tabular}{|l|c|c|c|c|c|}
\hline \multicolumn{5}{|c|}{ CRITERIOS } & \multicolumn{5}{|c|}{ PROVEEDORES } \\
\cline { 2 - 6 } & AMD & $\begin{array}{c}\text { DR } \\
\text { GLOBAL } \\
\text { SERVICE }\end{array}$ & $\begin{array}{c}\text { INDUSTRIAS } \\
\text { ALFA }\end{array}$ & $\begin{array}{c}\text { SUMIFLOR } \\
\text { CIA LTDA }\end{array}$ & $\begin{array}{c}\text { GAVETA } \\
\text { FLOR } \\
\text { CIA } \\
\text { LTDA }\end{array}$ \\
\hline Costo & 40 & 40 & 30 & 20 & 30 \\
\hline $\begin{array}{l}\text { Caracteristicas } \\
\text { Técnicas }\end{array}$ & 30 & 0 & 20 & 30 & 10 \\
\hline Garantia & 10 & 20 & 10 & 0 & 10 \\
\hline Expericncia & 5 & 10 & 10 & 0 & 5 \\
\hline TOTAL & $\mathbf{8 5}$ & $\mathbf{7 0}$ & 70 & $\mathbf{5 0}$ & $\mathbf{5 5}$ \\
\hline
\end{tabular}

Fuente: Elaboración propia

\section{- Proceso de evaluación.}

Después de haber seleccionado los proveedores, éstos se someten a una evaluación tomando en cuenta dos aspectos fundamentales: "plazo de entrega" y "calidad del bien y/o servicio".

Como premisa, las evaluaciones permiten dar seguimiento al proceder de los proveedores, determinar la eficiencia de los mismos y tomar medidas cuando sea necesario.

Tabla 9. Matriz de Evaluación de proveedores

\begin{tabular}{|c|c|c|c|c|c|c|c|}
\hline \multirow{3}{*}{$\begin{array}{l}\text { Aspectos } \\
\text { relevantes }\end{array}$} & \multirow{3}{*}{ 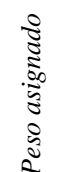 } & \multicolumn{6}{|c|}{ PROVEEDORES } \\
\hline & & \multicolumn{2}{|c|}{ PROVEEDOR AMD } & \multicolumn{2}{|c|}{$\begin{array}{l}\text { PROVEEDOR DR GLOBAL } \\
\text { SERVICE }\end{array}$} & \multicolumn{2}{|c|}{$\begin{array}{l}\text { PROVEEDOR } \\
\text { INDUSTRIAS ALFA }\end{array}$} \\
\hline & & Calificación & $\begin{array}{l}\text { Calificación } \\
\text { ponderada }\end{array}$ & Calificación & $\begin{array}{l}\text { Calificación } \\
\text { ponderada }\end{array}$ & Calificación & $\begin{array}{l}\text { Calificación } \\
\text { ponderada }\end{array}$ \\
\hline $\begin{array}{l}\text { Plazo de } \\
\text { entrega }\end{array}$ & $\widehat{0,35}$ & 5 & 1,75 & 1 & 0,35 & 3 & 1,05 \\
\hline $\begin{array}{l}\text { calidad del bien } \\
\text { y/o servicio }\end{array}$ & 0,65 & 3 & 1,95 & 3 & 1,95 & 5 & 3,25 \\
\hline Total & & & 3,7 & & 2,3 & & 4,3 \\
\hline
\end{tabular}

Fuente: Elaboración propia

\section{Indicadores de Productividad}

Adicional a los indicadores detallados anteriormente, el modelo de calidad se fundamenta en indicadores clave del proceso productivo que permiten evaluar, de forma directa, el desempeño de la productividad considerando recursos importantes como la mano de obra expresadas en horas hombre, disponibilidad de horas de trabajo y producción real, entre otras. 
Tabla 10. Datos mensuales de producción

\begin{tabular}{|l|c|c|}
\hline \multicolumn{1}{|c|}{ Datos } & Unidades & Valor \\
\hline A. Trabajadores & $\#$ & 12 \\
\hline B. Dias planificados & Dias & 20 \\
\hline C. Dias reales & Dias & 22 \\
\hline D. Joraada laboral & h & 8 \\
\hline E. Producción real & Ton & 250 \\
\hline F. Producción planificada & Ton & 280 \\
\hline G. Valor unitario & S/Ton & $\mathbf{8 0}$ \\
\hline H. Costo unitario & STon & $\mathbf{5 0}$ \\
\hline
\end{tabular}

Fuente: Elaboración propia

\section{Desarrollo.}

\section{- Productividad semanal}

$\mathrm{T}_{(\mathrm{h}-\mathrm{h}) \text { real }}=12$ hombres $* 176$ horas $=2112 \mathrm{~h}$

$\mathrm{T}_{(\mathrm{h}-\mathrm{h}) \text { planif }}=12$ hombres $* 160$ horas $=1920 \mathrm{~h}$

Productividad $_{(\mathrm{h}-\mathrm{h}) \text { real }}=\frac{\text { Producción real }}{\mathrm{T}_{(\mathrm{h}-\mathrm{h}) \text { real }}}=0,1184 \frac{\mathrm{Ton}}{\mathrm{h}-\mathrm{h}}$

Productividad semanal $=$ Productividad $_{(\mathrm{h}-\mathrm{h}) \text { real }} * 40 \mathrm{~h}=\mathbf{4}, \mathbf{7} \frac{\text { Ton }}{\mathbf{4 0 h}-\mathbf{h}}$

\section{- Eficacia de producción (\%)}

Eficacia de producción $=\frac{\text { Productividad }_{(\mathrm{h}-\mathrm{h}) \text { real }}}{\text { Productividad }_{(\mathrm{h}-\mathrm{h}) \text { planif }}} * 100$

Eficacia de producción $=81,17 \%$

\section{- Costo de operación (\$/h.h):}

$\begin{aligned} \text { Costo de operación } & =\frac{\text { Costo unitario }\left(\frac{\$}{\text { Ton }}\right) * \text { Producción real(Ton) }}{\text { Tiempo }_{(\text {h.h }) \text { real }}} \\ \text { Costo de operación } & =5,92 \frac{\$}{\mathrm{~h}-\mathrm{h}}\end{aligned}$

- Utilidad de venta (\%)

Utilidad de venta $(\%)=\frac{\text { Producción real } * \text { Valor unitario }- \text { costo operación } * \text { Total }(\mathrm{h}-\mathrm{h}) \text { real }}{\text { Producción real } * \text { Valor unitario }}$ Utilidad en venta $=37,5 \%$ 
- Utilidad respecto a costos (\%):

Utilidad de venta $(\%)=\frac{\text { Producción real } * \text { Valor unitario }- \text { costo operación } * \text { Total }(\mathrm{h}-\mathrm{h}) \text { real }}{\text { costo operación } * \text { Total }(\mathrm{h}-\mathrm{h}) \text { real }}$

Utilidad respecto a costos $=60 \%$

- Costo sobre ventas $(\%)$

$$
\begin{gathered}
\text { Costo sobre ventas }=\frac{\text { Costo unitario }(\$)}{\text { Precio unitario }(\$)} * 100 \\
\text { Costo sobre ventas }=62,5 \%
\end{gathered}
$$

\section{Control Estadístico de Procesos y Gráficas de Control para medir la Satisfacción del Cliente.}

A partir de la aplicación de una encuesta para medir el grado de satisfacción del cliente y utilizando la escala de Likert se obtuvieron los siguientes datos

Tabla 12. Datos satisfacción del cliente $(\mathrm{LCl}=4)$

\begin{tabular}{|c|c|c|}
\hline Aato & Mes & Satisfacción sobre 5.9 \\
\hline 2012 & Encro & 4.8 \\
\hline 2012 & Febrero & 4.5 \\
\hline 2012 & Marzo & 4.6 \\
\hline 2012 & Abril & 4.8 \\
\hline 2012 & Mayo & 4.7 \\
\hline 2012 & Junio & 4.8 \\
\hline 2012 & Julio & 4.5 \\
\hline 2012 & Agosto & 4.8 \\
\hline 2012 & Septiembre & 4.6 \\
\hline 2012 & Octubre & 4.6 \\
\hline 2012 & Noviember & 4.6 \\
\hline 2012 & Diciembre & 4.6 \\
\hline 2013 & Enero & 4.5 \\
\hline 2013 & Febrero & 4.5 \\
\hline 2013 & Marro & 4.8 \\
\hline 2013 & Abril & 4.7 \\
\hline 2013 & Mayo & 4.8 \\
\hline 2013 & Junio & 4.9 \\
\hline 2013 & Julio & 4.6 \\
\hline 2013 & Agosto & 4.8 \\
\hline 2013 & Septiembre & 4.6 \\
\hline 2013 & Octubre & 4.7 \\
\hline 2013 & Noviembre & 4.8 \\
\hline 2013 & Diciembre & 4.8 \\
\hline & & \\
\hline
\end{tabular}

Fuente: Elaboración propia 
- Análisis de los datos.

El conjunto de datos de la Tabla 12, permite suponer que se refieren a valores promedios obtenidos de una muestra de clientes seleccionada en cada mes. Por lo tanto los gráficos de control apropiados para representar éstos datos son gráficos de control de observaciones individuales y rangos móviles (Cuatrecasas, 2000).

Estos gráficos de control se emplean cuando se dispone de pocos datos o cuando no se tiene datos agrupados en forma de muestras como es el caso. (Cuatrecasas, 2000).

La ventaja de utilizar los dos gráficos es que el primer gráfico permite determinar la evolución del centrado de la característica (satisfacción del cliente) y la variabilidad asociada al mismo, mientras que el segundo gráfico permite medir la dispersión de la característica.

Para la aplicación de éstos gráficos, se requiere comprobar que los datos individuales siguen una distribución normal. Statfit, es una herramienta que permite determinar el tipo de distribución asociado al conjunto de datos. (Garcia E, 2013)

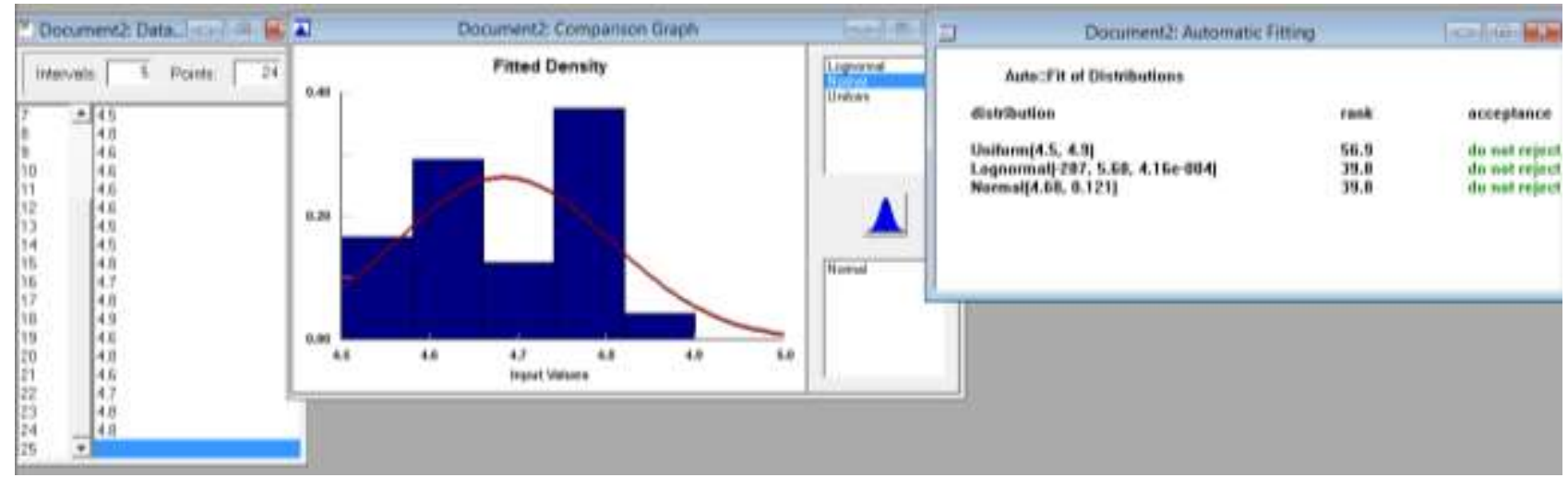

Gráfico 1. Resultados de Stat:fit

Fuente: Elaboración propia

De acuerdo al gráfico, se observa que el conjunto de datos siguen una distribución normal por lo tanto se puede aplicar los gráficos de control de observaciones individuales y rangos móviles.

Tabla 13. Rangos móviles

\begin{tabular}{lccc}
\hline Año & Mes & Satisfacción sobre 5.0 & Rango móvil \\
\hline 2017 & Enero & $\mathbf{4 . 8}$ & \\
2017 & Febrero & $\mathbf{4 . 5}$ & 0.3 \\
2017 & Marzo & $\mathbf{4 . 6}$ & 0.1 \\
2017 & Abril & $\mathbf{4 . 8}$ & 0.2 \\
2017 & Mayo & $\mathbf{4 . 7}$ & 0.1 \\
2017 & Junio & $\mathbf{4 . 8}$ & 0.1 \\
2017 & Julio & $\mathbf{4 . 5}$ & 0.3 \\
\hline
\end{tabular}




\begin{tabular}{lccc}
\hline 2017 & Agosto & $\mathbf{4 . 8}$ & 0.3 \\
2017 & Septiembre & $\mathbf{4 . 6}$ & 0.2 \\
2017 & Octubre & $\mathbf{4 . 6}$ & 0 \\
2017 & Noviembre & $\mathbf{4 . 6}$ & 0 \\
2017 & Diciembre & $\mathbf{4 . 6}$ & 0 \\
2018 & Enero & $\mathbf{4 . 5}$ & 0.1 \\
2018 & Febrero & $\mathbf{4 . 5}$ & 0 \\
2018 & Marzo & $\mathbf{4 . 8}$ & 0.3 \\
2018 & Abril & $\mathbf{4 . 7}$ & 0.1 \\
2018 & Mayo & $\mathbf{4 . 8}$ & 0.1 \\
2018 & Junio & $\mathbf{4 . 9}$ & 0.1 \\
2018 & Julio & $\mathbf{4 . 6}$ & 0.3 \\
2018 & Agosto & $\mathbf{4 . 8}$ & 0.2 \\
2018 & Septiembre & $\mathbf{4 . 6}$ & 0.2 \\
2018 & Octubre & $\mathbf{4 . 7}$ & 0.1 \\
2018 & Noviembre & $\mathbf{4 . 8}$ & 0.1 \\
2018 & Diciembre & $\mathbf{4 . 8}$ & 0 \\
\hline
\end{tabular}

Fuente: Elaboración propia

a. Límites de control para el gráfico de observaciones individuales.

- Límite central: $\mathrm{LC}=\mathrm{x}_{\mathrm{m}}$

- Límite de control superior: $\mathrm{LCS}=\mathrm{x}_{\mathrm{m}}+\mathrm{E}_{2} * \mathrm{R}_{\mathrm{m}}$

Dónde:

- Límite de control inferior: $\quad \mathrm{LCI}=\mathrm{x}_{\mathrm{m}}-\mathrm{E}_{2} * \mathrm{R}_{\mathrm{m}}$

$\mathrm{x}_{\mathrm{m}}$ : es el promedio de las observaciones individuales

$\mathrm{R}_{\mathrm{m}}$ : es el promedio de los rangos móviles

$\mathrm{E}_{2}$ : Coeficiente tabulado

b. Límites de control para el gráfico de rangos móviles.

- Límite central: $\mathrm{LC}=\mathrm{R}_{\mathrm{m}}$

- Límite de control superior: $\quad \mathrm{LCS}=\mathrm{D}_{4} * \mathrm{R}_{\mathrm{m}}$

- Límite de control inferior: $\quad \mathrm{LCI}=\mathrm{D}_{3} * \mathrm{R}_{\mathrm{m}}$

- $\quad \mathrm{D}_{3}, \mathrm{D}_{4}$ : coeficientes tabulados

Para nuestro caso, los valores son:

$\left.\mathrm{x}_{\mathrm{m}}=4,683 \quad \mathrm{R}_{\mathrm{m}}=0,1391 \quad \mathrm{~s}=0,1239\right\}$

$\mathrm{n}=2$ (número de datos para el cálculo de los

rangos móviles)

$\mathrm{E}_{2}=2,660 \quad \mathrm{D}_{3}=0 \quad \mathrm{D}_{4}=3,267$ 
Tabla 14. Resultados: Límites de Control

\begin{tabular}{ccc}
\hline Limites de control & $\begin{array}{c}\text { Gráfico de } \\
\text { observaciones } \\
\text { individuales }\end{array}$ & $\begin{array}{c}\text { Gráfico de rangos } \\
\text { móviles }\end{array}$ \\
\hline $\boldsymbol{L C}$ & 4,683 & 0,139 \\
$\boldsymbol{L C S}$ & 5,053 & 0,454 \\
$\boldsymbol{L C I}$ & 4,313 & 0 \\
\hline
\end{tabular}

Fuente: Elaboración propia

Las gráficas de control para observaciones individuales y rangos móviles mostradas en el Gráfico 2 y diseñadas en minitab, indican que el proceso de atención al cliente es estable es decir, los clientes se sienten muy satisfechos con la atención recibida. La calificación representa el nivel de satisfacción promedio entre 4,31 y 5, valores que están incluso, por debajo de los límites de tolerancia establecidos.

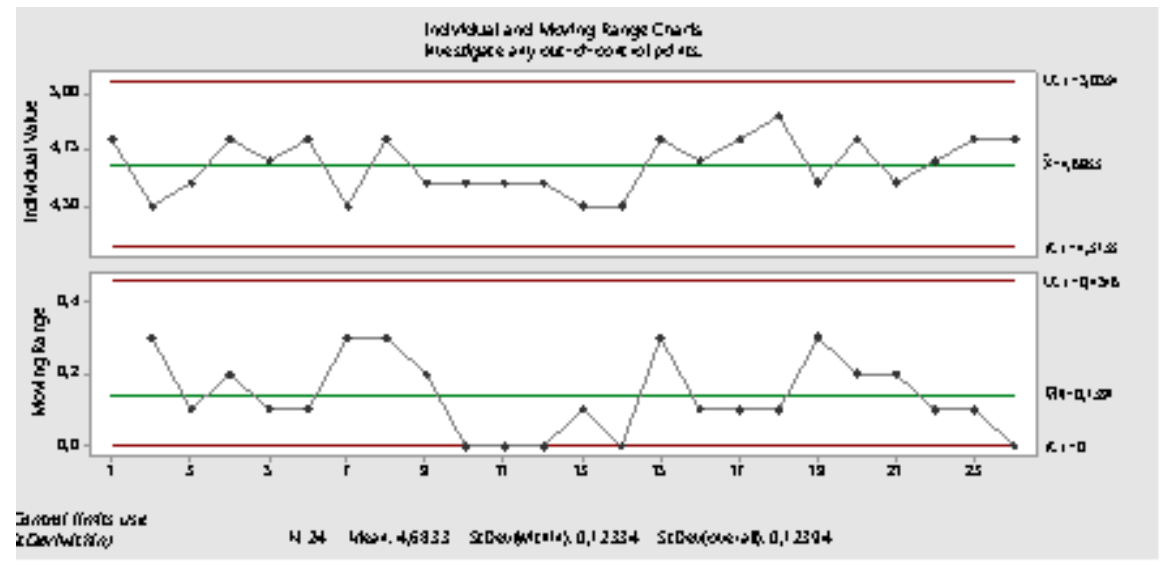

Gráfica 2. Gráficas de control para observaciones individuales y rangos móviles (I - MR) Fuente: Elaboración propia

No existen puntos atípicos en las gráficas de control, lo cual significa que el nivel de satisfacción del cliente solo se debe a causas comunes o aleatorias que actúan de una forma estable provocando una variabilidad regular aceptable. La función de distribución de probabilidad del conjunto de datos que originan éstas causas es la distribución normal.

Al no existir un patrón de comportamiento de los datos con la evolución del gráfico de control se concluye que no existen anomalías concretas en el proceso de servicio al cliente y por lo tanto garantiza que el proceso sólo está sometido a causas aleatorias de variabilidad. (Cuatrecasas, 2000) 
El diagrama de Pareto que se muestra en el Gráfico 3. indica que aproximadamente el $70 \%$ de los clientes en promedio califican la atención al cliente entre 4,6 y 4,8 sobre 5; resultados que indican que el personal está altamente calificado, así también que los procesos son efectivos y que contribuye con el desarrollo del negocio y sus actores.

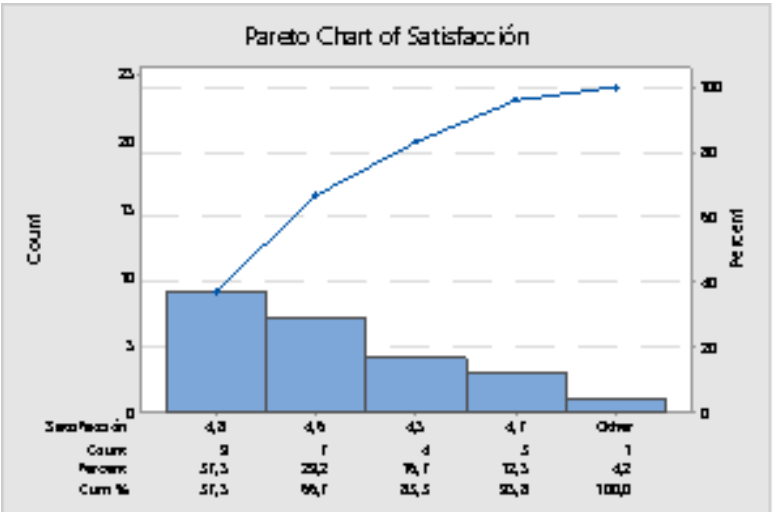

Gráfico 3. Diagrama de Pareto

Fuente: Elaboración propia

El Gráfico 4 muestra un histograma de frecuencias en el cual los datos se ajustan a una distribución normal, requisito para la aplicación de los modelos I - RM. El mismo resultado se obtuvo aplicando la herramienta Stat:fit, utilizado para determinar el tipo de distribución al cual pertenece el conjunto de datos.

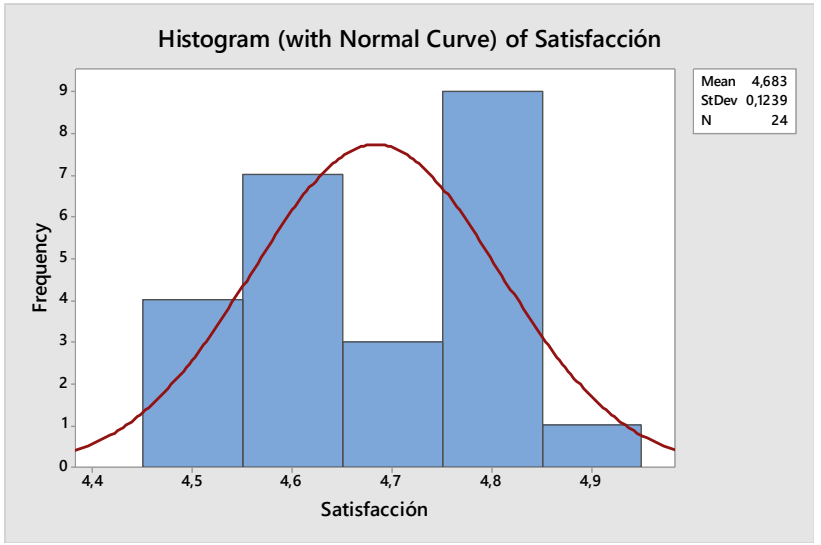

Gráfico 4. Histograma de frecuencias.

Fuente: Elaboración propia

Stat:fit muestra que los datos se ajustan a tres tipos de distribuciones como lo muestra la figura $\mathrm{N}^{\circ} 8$ y estos son: la uniforme y la log normal y normal, sin embargo, para el de caso de estudio, se selecciona la distribución normal, lo cual permite aplicar correctamente el tipo de gráfico de control. (Garcia E, 2013) 


\section{Capacidad de proceso $\left(C_{p}\right)$ :}

$$
\mathrm{C}_{\mathrm{p}}=\frac{\mathrm{TS}-\mathrm{TI}}{6 \mathrm{~s}}=\frac{5-4}{6 * 0,1239}=\mathbf{1}, \mathbf{3 4 5}
$$

\section{Índice de capacidad real $\left(\mathrm{C}_{\mathrm{pk}}\right)$ :}

$$
\begin{aligned}
& C_{\mathrm{pk}}=\text { Mín }\left[\frac{\mathrm{TS}-\mathrm{x}_{\mathrm{m}}}{3 \mathrm{~s}} ; \frac{\mathrm{x}_{\mathrm{m}}-\mathrm{TT}}{3 \mathrm{~s}}\right] \\
& =\left[\frac{5-4,683}{3 * 0,1239} ; \frac{4,683-4}{3 * 0,1239}\right] \\
& =[0,8516 ; 1,8377] \\
& =\mathbf{0}, \mathbf{8 5 1 6}
\end{aligned}
$$

La medición de la satisfacción del cliente es un proceso de máximos, puesto que el menor valor es 0 y el valor óptimo deseado es 5.

Para asegurar que el 99,7\% de los clientes están satisfechos, el intervalo que define los límites de tolerancia (LTS-LTI) debe ser $\geq$ a 6s, (Cuatrecasas, 2000), es decir debe cumplirse pues:

$$
\mathrm{C}_{\mathrm{p}}=\frac{\mathrm{LTS}-\mathrm{LTI}}{6 \mathrm{~s}} \geq 1
$$

Por lo tanto, según la tabla $\mathrm{N}^{\circ} 14$, mostrada anteriormente, nuestro límite de tolerancia inferior es 4 y nuestro límite de tolerancia superior es 5 .

El resultado obtenido de $C_{p}=\mathbf{1}, 345$ nos indica que la calificación de los clientes está 'satisfactoriamente dentro de los límites de tolerancia establecidos ya que $\mathrm{Cp}>1$, esto significa que existen muy pocos clientes insatisfechos; haciendo analogía a lo que menciona según Runger (2011) que cuando $\mathrm{Cp}=1$ la proporción de unidades no conformables es de 0,27\%, es decir 2700 clientes insatisfechos por millón.

El índice de capacidad real del proceso $\left(\mathrm{C}_{\mathrm{pk}}\right)$ toma en cuenta el hecho de que en la vida real la media no coincide con el valor central óptimo, calcula cuantas veces cabe la distribución descentrada en el intervalo de tolerancias (LTS - LTI). (Cuatrecasas, 2000).

En éste caso, por ser un proceso con característica de calidad cualitativa, nos interesa el índice de capacidad real inferior $\left(\mathrm{C}_{\mathrm{pk}_{\mathrm{s}}}\right)=0,8516$ ya que se espera que la media de los datos se acerque al valor optimo deseado. Debido a que el proceso no es capaz, A veces es necesario es necesario centrar un proceso para cumplir con los requisitos establecidos. Es decir, se debe ajustar el proceso de tal forma que la variabilidad y el centrado cumplan con las características deseadas. 


\section{Conclusiones.}

- El caso de estudio brinda la oportunidad para aplicar métodos de mejora enfocados a los procesos y que se pueden extender a procesos de similares características en las empresas agrícolas. Orienta el desarrollo de la capacidad para generar soluciones a los problemas con base en la interpretación de los resultados que conducen a formulaciones de decisiones adecuadas para lograr los objetivos planteados.

- Se han planteado metodologías, se ha propuesto indicadores de desempeño los cuales permiten medir el comportamiento de las variables en función de la implementación de las soluciones.

- La empresa florícola debe estar acorde con los lineamientos de la matriz productiva que buscan mejorar los procesos productivos con estrategias y tecnología innovadoras, a bajos costos y generando valores agregados a la producción para mejorar la calidad de la diversidad de rosas que comercializa y hacerlo con velocidad de respuesta rápida de modo que minimice la insatisfacción del cliente y, al contrario, lo haga fiel a la marca. Por lo tanto la empresa para ser competitiva, debe mejorar todos los procesos de la organización de forma integrada.

- Al aplicar los métodos que se han descrito en los diferentes literales, la empresa tendrá la posibilidad de dirigir mejor sus acciones para lograr sus objetivos planteados; mantener bajo control ciertos procesos críticos de la organización en cuanto a satisfacción del cliente e incremento de ventas, direccionando correctamente sus esfuerzos para conseguirlo.

- En la mayoría de los literales, al término de su desarrollo, se hace una breve descripción e interpretación de los resultados así como de conclusiones y que se relacionan con lo que se pretende buscar con el literal.

\section{Referencias bibliográficas.}

Berovides-Castellón, M., \& Michelena-Fernández, E. (2013). La gestión de la calidad en una empresa de pastas alimenticias. Ingeniería Industrial, XXXIV (3), 252-266.

Boletín Central de análisis sectorial de MIPYMES. FLACSO_ MIPRO. Centro de Investigaciones Económicas de la micro, pequeña y mediana empresa (pag. 7)

Carro, R., González D. (2010). Diseño y selección de procesos. Administración de las Operaciones. Facultad de Ciencias Económicas y Sociales, Universidad Nacional del Mar del Plata.

Caselles, J. M. (2008). Gerencia de Procesos. Obtenido de Gerencia de Procesos: http://gerenciaprocesos.comunidadcoomeva.com/blog/index.php?/categories/3-3Macroprocesos

Cuatrecasas, E. B. (2000). Seis Sigma Una iniciativa de Calidad Total. Barcelona: Ediciones Gestión 2000 
Lafuente, E.; Bayo, A.; García, M., «ISO-9000 Certification and Ownership Structure: Effects upon Firm Performance» British Journal of Management, 2009, vol. 36, no. 3, pp. 7-11, ISSN 4427-3264-5405

Gómez, O. (2011). Los costos y procesos de producción, opción estratégica de productividad y competitividad en la industria de confecciones infantiles de Bucaramanga. Revista EAN, (70), 167-180.

García E., H. G. (2013). Simulación y Análisis de sistemas con ProModel. México: Pearson.

Holgado, J. (s.f.). Seminario de Project Management. Obtenido de Seminario de Project Management: http://es.slideshare.net/JesusHolgado/seminario-pm-mundoproyectos

ICONTEC. (s.f.). Indicadores de Gestión. Obtenido de Indicadores de Gestión: http://www.pascualbravo.edu.co/pdf/calidad/indicadores.pdf

ISO 9001:2000 "Sistemas de Gestión de Calidad. Requisitos". (s.f.). Obtenido de ISO 9001:2000 "Sistemas de Gestión de Calidad. Requisitos": http://iso9001calidad.com/iso-9001-2000-sistemas-gestion-calidad-requisitos21.html

Pereiro, J. (2005). Gestión de las compras y la evaluación de proveedores en ISO 9001:2000. Obtenido de Gestión de las compras y la evaluación de proveedores en ISO 9001:2000:

http://www.portalcalidad.com/articulos/56gestion_compras_y_evaluacion_proveedo res_iso_9001:2000

Render, J. H. (2009). Principios de Administración de Operaciones. México: Pearson.

Rosales, R. (2008). ADMINISTRACIÓN GERENCIA Y GESTIÓN. Obtenido de ADMINISTRACIÓN GERENCIA Y GESTIÓN: http://facsoagg2007.blogspot.com/2008/01/ricardo-rosales-analisis-sobre-misiny.html

Runger, D. C. (2011). Probabilidad y Estadísitca aplicada a la Ingeniería. México: Limusa Wiley .

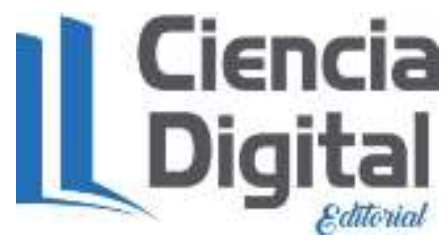




\section{PARA CITAR EL ARTÍCULO INDEXADO.}

Acosta J., Bonilla S., Santiago L., Jácome E. \& Miño G. (2019). Modelo de gestión de calidad para empresas agroindustriales. Caso de estudio: florícola. Revista electrónica Ciencia Digital 3(2), 483-507. Recuperado desde:

http://cienciadigital.org/revistacienciadigital2/index.php/CienciaDigital/article/view/446/1008

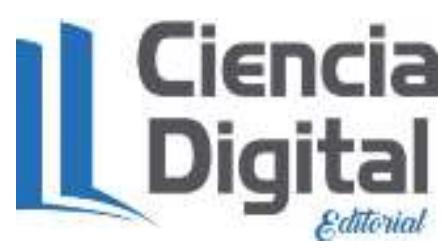

El artículo que se publica es de exclusiva responsabilidad de los autores y no necesariamente reflejan el pensamiento de la Revista Ciencia Digital.

El artículo queda en propiedad de la revista y, por tanto, su publicación parcial y/o total en otro medio tiene que ser autorizado por el director de la Revista Ciencia Digital.
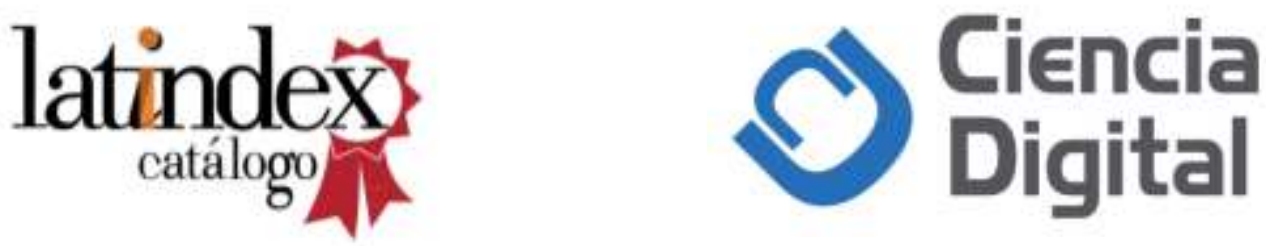